\title{
Solar UV Variations During the Decline of Cycle 23
}

\author{
Matthew T. DeLand ${ }^{\mathrm{a}}$ (matthew.deland@ssaihq.com), Richard P. Cebula ${ }^{\mathrm{a}}$ \\ ${ }^{a}$ Science Systems and Applications, Inc., Lanham, MD 20706 USA
}

\begin{abstract}
Characterization of temporal and spectral variations in solar ultraviolet irradiance over a solar cycle is essential for understanding the forcing of Earth's atmosphere and climate. Satellite measurements of solar UV variability for solar cycles 21,22 , and 23 show consistent solar cycle irradiance changes at key wavelengths (e.g. $205 \mathrm{~nm}, 250 \mathrm{~nm}$ ) within instrumental uncertainties. All historical data sets also show the same relative spectral dependence for both short-term (rotational) and long-term (solar cycle) variations. Empirical solar irradiance models also produce long-term solar UV variations that agree well with observational data. Recent UV irradiance data from the Solar Radiation and Climate Experiment (SORCE) Spectral Irradiance Monitor (SIM) and Solar Stellar Irradiance Comparison Experiment (SOLSTICE) instruments covering the declining phase of Cycle 23 present a different picture of long-term solar variations from previous results. Time series of SIM and SOLSTICE spectral irradiance data between 2003 and 2007 show solar variations that greatly exceed both previous measurements and predicted irradiance changes over this period, and the spectral dependence of the SIM and SOLSTICE variations during these years do not show features expected from solar physics theory. The use of SORCE irradiance variations in atmospheric models yields substantially different middle atmosphere ozone responses in both magnitude and vertical structure. However, short-term solar variability derived from SIM and SOLSTICE UV irradiance data is consistent with concurrent solar UV measurements from other instruments, as well as with previous results, suggesting no
\end{abstract}


change in solar physics. Our analysis of short-term solar variability is much less sensitive to residual instrument response changes than the observations of long-term variations. The SORCE long-term UV results can be explained by undercorrection of instrument response changes during the first few years of measurements, rather than requiring an unexpected change in the physical behavior of the Sun.

\section{Introduction.}

Solar ultraviolet radiation $(\lambda<400 \mathrm{~nm})$ is the primary forcing mechanism for the photochemistry, heating, and dynamics of the Earth's atmosphere. The altitude at which solar energy is deposited varies with wavelength, as shown in Figure 1. Thus, accurate knowledge of both temporal and spectral variations in solar UV irradiance is necessary to model and understand atmospheric behavior. Figure 1 also shows that only space-based measurements can gather this information on a regular basis. Solar UV measurements have been made by at least seventeen satellite instruments since 1978, as shown in Figure 2. Significant data sets available from these measurements include Solar Mesosphere Explorer (SME) (Rottman, 1988), Nimbus-7 Solar Backscatter Ultraviolet spectrometer (SBUV) (DeLand and Cebula, 2001), NOAA-9 SBUV model 2 (SBUV/2) (DeLand et al., 2004), NOAA-11 SBUV/2 (DeLand and Cebula, 1998), Shuttle SBUV (SSBUV) (Cebula et al., 1996), Upper Atmosphere Research Satellite (UARS) Solar Ultraviolet Spectral Irradiance Monitor (SUSIM) (Floyd et al., 2003), and UARS Solar Stellar Irradiance Comparison Experiment (SOLSTICE) (Rottman et al., 2001). Additional historical information about many of these measurements is given in DeLand and Cebula (2008).

The Solar Radiation and Climate Experiment (SORCE) spacecraft, launched in December 2003, carries two instruments dedicated to continuing the solar UV irradiance data record. The SOLSTICE II instrument is similar in design to the UARS SOLSTICE instrument, although its 
spectral coverage is limited to $115-310 \mathrm{~nm}$ (McClintock et al., 2005). The Spectral Irradiance Monitor (SIM) is the first space-based instrument to continuously measure solar spectral irradiance variations across the ultraviolet, visible, and near infrared spectral regions, and covers the wavelength range 200-2400 $\mathrm{nm}$ (Harder et al., 2005). Observations from SIM suggest that from 2004 to 2007, during the declining phase of solar cycle 23, solar UV irradiance decreased by a larger factor than observed during 30 years of prior UV irradiance measurements (Harder et al., 2009). The integrated irradiance change between $200-400 \mathrm{~nm}$ over this period derived from SIM observations is a factor of 10 larger than the corresponding irradiance change calculated by the model of Lean et al. (2005).

The SIM solar UV irradiance variations, when used in atmospheric models, also have a dramatic effect on calculated stratospheric ozone changes during decreasing solar activity compared to currently accepted results. Using the SIM data results in model-predicted ozone decreases at $35 \mathrm{~km}$ that are a factor of three greater than those predicted using standard estimates of solar variability, and unexpected ozone increases are predicted above $45 \mathrm{~km}$ (Haigh et al., 2010; Merkel et al., 2011). SORCE SOLSTICE irradiance changes between 200-300 nm shown in Haigh et al. (2010), while smaller than those reported by SIM, are still substantially larger than current models predict. Simultaneously, the solar spectral irradiance reported by SIM increased at visible and infrared wavelengths, even as solar activity and total solar irradiance decreased (Harder et al., 2009). Incorporating these SIM spectral irradiance changes into climate model calculations suggests "the effects of solar variability on temperature throughout the atmosphere may be contrary to current expectations" (Haigh et al., 2010), with the conclusion that higher solar activity cools, rather than warms, the Earth. Further discussion of the reported SIM results at visible and near-IR wavelengths, and the implications of these results for climate 
models, are given in Lean and DeLand (How does the Sun's spectrum vary?, submitted to Journal of Climate, 2011).

This paper evaluates the accuracy of reported solar UV irradiance changes during the declining phase of Cycle 23. Historical evidence gives a consistent picture of the magnitude and spectral dependence of UV irradiance changes over a solar cycle. Multiple solar UV proxy data sets show that the most recent solar minimum was not significantly lower in magnitude than previous solar minima. We show that the SORCE measurements during Cycle 23 fall well outside the range determined by the 30 -year observational record and model calculations, with spectral variations that do not agree with solar physics theory. However, the SORCE irradiance results can be explained by undercorrection of instrument response changes during the first few years of on-orbit measurements. Analysis of short-term solar UV variations derived from SORCE data are consistent with concurrent irradiance measurements from UARS SUSIM and NOAA-17 SBUV/2, and produce irradiance scaling factors that agree well with previous results. Recent efforts to extend atmospheric model studies based on SORCE UV variations to a complete solar cycle do not address their differences with the published solar UV data record. Thus, we conclude that the solar UV variations presented in Harder et al. (2009) and Haigh et al. (2010) are incorrect, and that the long-term calibrations of the SORCE SIM and SORCE SOLSTICE instruments need to be revised.

\section{Historic Solar UV Variability - Temporal.}

A key question for many studies of solar UV irradiance is to understand the magnitude of irradiance variations over the solar cycle, which has significant spectral dependence and can vary from cycle to cycle. We discuss the temporal variation at selected wavelengths in this section, 
and address the observed spectral variation in more detail in Section 3. While long-term UV irradiance variation results are available from many instruments, most of these measurements only covered one phase of a single solar cycle (either increasing or decreasing) to evaluate the difference between maximum and minimum solar activity. Variations at wavelengths such as $205 \mathrm{~nm}$ and $250 \mathrm{~nm}$ have been quoted in previous work because of their importance to middle atmosphere photochemistry. Published estimates of solar cycle variations at these wavelengths, including the portion of the solar cycle covered by each data set, are listed in Table 1.

Table 1. Observations of solar cycle UV irradiance variability (maximum to minimum).

\begin{tabular}{|l|l|l|l|l|}
\hline Instrument & $\mathbf{A F}(\mathbf{2 0 5} \mathbf{~ n m})$ & $\mathbf{\Delta F}(\mathbf{2 5 0} \mathbf{n m})$ & Solar cycle and & Reference \\
\hline SME & $6( \pm 4) \%$ & $3( \pm 4) \%$ & Cycle 21 desc. & Rottman (1988) \\
\hline Nimbus-7 SBUV & $8.3( \pm 2.6) \%$ & $4.9( \pm 1.8) \%$ & Cycle 21 desc. & DeLand and Cebula \\
& & & & $(2001)$ \\
\hline NOAA-9 SBUV/2 & $9.3( \pm 2.3) \%$ & $5.7( \pm 1.8) \%$ & Cycle 22 complete & DeLand et al. (2004) \\
\hline NOAA-11 SBUV/2 & $7.0( \pm 1.8) \%$ & $3.5( \pm 1.8) \%$ & Cycle 22 desc. & DeLand and Cebula \\
\hline UARS SUSIM & $6( \pm 2) \%$ & $4( \pm 2) \%$ & Cycle 22 desc. & Floyd et al. (2003) \\
\hline UARS SUSIM & $4.5( \pm 2) \%$ & $3.5( \pm 2) \%$ & Cycle 23 asc. & Floyd et al. (2003) \\
\hline UARS SOLSTICE & $6( \pm 2) \%$ & $3( \pm 2) \%$ & Cycle 22 desc. & Rottman et al. (2001) \\
\hline
\end{tabular}


These results are quite self-consistent when quoted uncertainties in the irradiance data sets and real inter-cycle differences in solar maximum activity are considered. DeLand and Cebula (2008) created a composite UV irradiance data product covering $1978-2005$ by combining measurements from all of the instruments listed here. Their results showed no significant difference between the UV irradiance levels for the solar activity minimum between Cycles 2122 and the minimum between Cycles 22-23.

Solar irradiance values can also be calculated using physically-based models and proxy indexes, such as the Mg II index (Heath and Schlesinger, 1986), that represent solar UV activity. One example is the model of Lean et al. (2005), now known as the Naval Research Laboratory Solar Spectral Irradiance (NRLSSI) model. This model uses a linear combination of empirically determined parameterizations for facular brightening and sunspot darkening to calculate spectrally varying solar UV irradiances. The Mg II index and white light solar images are used as proxies for chromospheric and photospheric variations, respectively. Irradiance time series from this model were compared to the composite UV irradiance data set by DeLand and Cebula (2008). The long-term agreement between these data sets is within $\sim 2 \%$ at $200-205 \mathrm{~nm}$ and $\sim 1 \%$ at $240-245 \mathrm{~nm}$, indicating that the model representation of long-term solar variations is consistent with observations.

Efforts to extend the quantitative determination of solar UV irradiance variations beyond a single solar cycle are complicated by the limited overlap between individual data sets and the interpretation of inter-instrument (spectrally varying) absolute calibration differences. DeLand and Cebula (2008) discuss these issues in more detail. These challenges mean that proxy indexes of solar UV activity are particularly important for evaluating differences between solar cycles. Figure 3 shows time series of three different solar UV proxy data sets (Mg II core-to-wing index, 
$10.7 \mathrm{~cm}$ radio flux, $\mathrm{Ca} \mathrm{II} \mathrm{K}$ index) covering the last three solar cycles. Comparing one-year averages of each data set during solar minimum periods, we find that the Cycle 23 minimum (2008.5-2009.5) is slightly lower in absolute value than the previous minima. In each case, though, these differences $\left(0.1 \%\right.$ in $\mathrm{Mg} \mathrm{II}, \sim 6 \%$ in $\mathrm{F}_{10.7}$ and $\left.\mathrm{Ca} \mathrm{II} \mathrm{K}\right)$ are a factor of 50 less than the amplitude of Cycle 23 calculated from the average index value during solar maximum (2001.5-2002.5). Thus, the UV proxy data sets do not indicate a significant difference between the Cycle 23 minimum and previous solar minima.

Based on the UV proxy data sets and total solar irradiance variations, we estimate that the limited time period between May 2004 and November 2007 discussed by Haigh et al. (2010) represents approximately one-third of the maximum to minimum dynamic range of Cycle 23. Figure 4 shows time series comparisons between the SORCE SIM data and the composite UV data record of DeLand and Cebula (2008) for four spectral bands. The SIM data presented here have been restricted to a shortest wavelength of $240 \mathrm{~nm}$ [J. Harder, personal communication], rather than the limit of $200 \mathrm{~nm}$ shown in Haigh et al. (2010) and Ball et al. (2011) or the limit of $220 \mathrm{~nm}$ shown in Harder et al. (2010). The SIM time series have been normalized to the composite data in April 2004 in each panel for comparison. At 240-245 nm and 270-275 nm, the SIM variations over a limited time period equal or exceed previously observed irradiance changes over a complete solar cycle. The SIM results thus imply a much larger solar cycle amplitude at these wavelengths than any previous results. For comparison with Table 1, the estimated solar cycle variation from these data at $250 \mathrm{~nm}$ is $11.4( \pm 0.5) \%$, where the long-term uncertainty estimate is taken from Merkel et al. (2011). While the long-term uncertainty of the composite data at $315-320 \mathrm{~nm}$ and $380-385 \mathrm{~nm}$ is typically larger than the variations shown in Figures $4(\mathrm{c})$ and $4(\mathrm{~d})$, we note that the long-term calibration and signal-to-noise ratio of the 
UARS SUSIM data (beginning December 1991 in the composite product) is better than the SBUV and SBUV/2 data used prior to that date. The SIM data show a rapid decrease at $315-320$ $\mathrm{nm}$ that is not reflected in the SUSIM data. The $380-385 \mathrm{~nm}$ band includes a Mg I Fraunhofer line at $382.94 \mathrm{~nm}$, so we believe that the modest solar cycle signature visible in the SUSIM data set in Figure 4(d) between 1997-2004 may be real.

Large temporal differences are also seen during the 2004-2007 time period between spectrally resolved SORCE SOLSTICE data and the composite UV data record, as shown in Figure 5. The SOLSTICE time series have been normalized to the composite data in September 2003 in each panel for comparison. The SOLSTICE data show a consistent solar minimum level with the composite data set at $185-190 \mathrm{~nm}$, but produce a significantly lower minimum level at 200-205 nm. At 230-235 nm, the SOLSTICE irradiance changes over a partial solar cycle are substantially larger than previous solar cycle variations. At $290-295 \mathrm{~nm}$, the SOLSTICE data show a continuous decrease with no evidence of solar minimum values in 2007-2009. This behavior is consistent with uncorrected instrument response changes. Extrapolating the SOLSTICE variations over a full solar cycle gives an estimated amplitude of $15.2( \pm 0.5) \%$ at 205 $\mathrm{nm}$ and $7.5( \pm 0.5) \%$ at $250 \mathrm{~nm}$, with uncertainty estimates taken from Snow et al. (2010).

Lockwood (2011) presents extensive analysis of the overlap period between UARS SUSIM and SORCE SOLSTICE data from May 2003 to August 2005, considering only the wavelength range 156-208 $\mathrm{nm}$. He evaluates multiple methods for combining these data sets, ranging from a simple zero-level offset adjustment to a more complex approach that varies both offset and relative instrument sensitivity with time, and assuming that either SUSIM or SOLSTICE is correct. He prefers the idea that SORCE SOLSTICE is correct based on having "higher levels of in-flight calibration available", although there is little discussion of why the SUSIM in-flight 
calibration (see Floyd et al. (1998) for detailed discussion) might be lacking. Using this assumption to merge the two data sets, Lockwood (2011) finds lower irradiance values during the Cycle 23 minimum only for wavelengths greater than $195 \mathrm{~nm}$. This is consistent with the results shown in Figure 5(a), where only an absolute adjustment was applied to the SORCE data.

Ball et al. (2011) present extensive comparisons between the Spectral and Total Irradiance Reconstruction (SATIRE) model and observations from SORCE TIM and SORCE SIM. The satellite era version of this model, denoted by SATIRE-S, determines spectral irradiance variations over the wavelength range $200-160,000 \mathrm{~nm}$ based on changes in the distribution of solar surface magnetic features. They found that SATIRE-S integrated irradiance values reproduced TIM TSI data very well, using the same value for the model's free parameter as determined from previous comparisons with UARS SUSIM and UARS SOLSTICE data. However, they also found that SIM data integrated over 201-300 nm decreased by a factor of 5 more than SATIRE-S calculations for 2004-2007, reaching solar minimum more than 1 year before the minimum in sunspot number. The integrated SIM data show a subsequent increase through October 2009 that equals the full solar cycle 22 variation as observed by UARS SUSIM. Ball et al. (2011) were unable to adjust the model parameters to match the SIM observations, and noted that even these unsuccessful attempts worsened their agreement in TSI reconstruction and rotational variability analyses. They also observed that the largest changes in SIM data occurred during the first two years of operation, when it is most difficult to maintain sensor radiometric stability, and when rapid sensitivity changes are most likely to occur.

\section{Historic Solar UV Variability - Spectral.}


The wavelengths used in Table 1 to illustrate solar cycle amplitude are representative of broader spectral regions where the physics of the solar atmosphere leads to distinctive structure (Foukal, 1990). Figure 6(a) shows the spectral dependence of solar cycle variation over 190-400 $\mathrm{nm}$ as observed for Cycle 22 by NOAA-9 SBUV/2, as observed for Cycle 23 by UARS SUSIM, and as calculated for Cycle 22 by the NRLSSI model. All data are binned to $1 \mathrm{~nm}$ sampling. Features such as the Al ionization edge at $207 \mathrm{~nm}$, the $\mathrm{Mg}$ ionization edge at $261 \mathrm{~nm}$, the $\mathrm{Mg} \mathrm{II}$ Fraunhofer line at $280 \mathrm{~nm}$, and the $\mathrm{Mg}$ I Fraunhofer line at $285 \mathrm{~nm}$ are clearly visible. These features are consistently present in well-calibrated observational data sets and model calculations. Figure 6(b) shows the reported solar change over 190-400 nm between May 2004 and November 2007 for the NRLSSI model, SORCE SIM measurements, and SORCE SOLSTICE measurements. This comparison is similar to Figure 1 of Haigh et al. (2010), but with solar variation expressed as a percentage change rather than in absolute irradiance units. Note that while the magnitude of the NRLSSI variations in this figure are much smaller than the solar cycle values shown in Figure 6(a) because of the time period selected, covering approximately one-third of the solar cycle amplitude, the same characteristic spectral dependence is observed. In contrast, the SIM data show a monotonic increase in reported irradiance change from $360 \mathrm{~nm}$ down to $240 \mathrm{~nm}$, with a change in the wavelength-dependent slope at $285 \mathrm{~nm}$. The magnitude of the SIM changes is a factor of three or more greater than the NRLSSI changes below $320 \mathrm{~nm}$. These larger values are consistent with an undercorrection of the SIM instrument response degradation during the declining phase of Cycle 23. SORCE SOLSTICE data in Figure 6(b) are consistent with SIM results between $260-290 \mathrm{~nm}$, but also show large peaks between 185-210 $\mathrm{nm}$ and 215-245 $\mathrm{nm}$ that are not observed in other measurements. These peaks are also consistent with undercorrected instrument degradation. Snow et al. (2010) suggest that a similar 
undercorrection is responsible for discrepancies at 140-155 $\mathrm{nm}$ in comparisons between UARS SOLSTICE and SORCE SOLSTICE solar minimum irradiance values.

\section{Instrument Calibration.}

Satellite remote sensing instruments frequently experience significant response changes that, if not properly characterized, can be mistakenly interpreted as real geophysical variability. A specific example of this situation occurred for the Nimbus-7 SBUV instrument measuring stratospheric ozone, where the instrument design made it difficult to determine the separation of response changes between optical components and the solar diffuser plate. While initial studies using Nimbus-7 SBUV data indicated more rapid atmospheric ozone changes than expected due to increasing concentrations of chlorofluorocarbons (Heath, 1988), further analysis showed that an alternate model of instrument behavior gave SBUV ozone trends that were much more consistent with results from multiple independent data sources (Herman et al., 1990).

Instruments that measure solar UV irradiance are particularly susceptible to on-orbit degradation, because the energetic UV photons will photolyze contaminants on the optical surfaces. These changes often show an exponential time dependence, with the most rapid changes occurring early in the instrument's lifetime. The magnitude of the overall sensitivity decrease after just a few years is frequently greater than estimated long-term solar activity variations during the same interval. Examples of such uncorrected sensitivity changes are shown in the literature for Nimbus-7 SBUV (DeLand and Cebula, 2001), NOAA-9 SBUV/2 (Ahmad et al., 1994; DeLand et al., 2004), NOAA-11 SBUV/2 (Hilsenrath et al., 1995; Cebula and DeLand, 1998), UARS SOLSTICE (Rottman et al., 2001), and UARS SUSIM (Floyd et al., 
1998). These rapid changes thus complicate the determination of real solar UV variability from the satellite measurements.

The SORCE solar UV instruments have also experienced significant degradation in space. Snow et al. (2005) show SORCE SOLSTICE sensitivity changes derived from stellar measurements of $7 \%$ at $155 \mathrm{~nm}$ during the first two years of operation, although changes at longer wavelengths $(180-310 \mathrm{~nm})$ were reported to be $2 \%$ or less. The overall stellar degradation change at $155 \mathrm{~nm}$ has increased to $\sim 12 \%$ through early 2011 [M. Snow, private communication], which is a factor of three less degradation than the cumulative UARS SOLSTICE degradation (including one-time events) over a similar time period.

Harder et al. (2010) state that the response of the SIM A (primary) spectrometer has degraded by $27.5 \%$ at $280 \mathrm{~nm}$ during the first 2000 days ( $\sim 5.5$ years) of operation, decreasing in magnitude to only $1.27 \%$ at $656 \mathrm{~nm}$ during the same period. The supplemental material to Harder et al. (2009) defines the change in SIM transmission as:

$\mathrm{T}(\lambda, t)=\mathrm{T}_{0} e^{-\tau(\lambda, \mathrm{t}-\mathrm{t} 0)}$

where $\tau$ can be separated into orthogonal terms such that

$$
\tau(\lambda, t)=\kappa(\lambda) \cdot C(t)
$$

$\kappa(\lambda)$ is interpreted as the opacity of the absorbing surface material on the instrument prism, and $\mathrm{C}(\mathrm{t})$ is interpreted as the accumulated column surface density of the material. Figures S5 and S2 in the on-line supplement to Harder et al. (2009) show the spectral and temporal dependence of 
these parameters. The SIM instrument also uses a secondary (B) spectrometer for references, whose overall exposure time is approximately $22 \%$ of that accumulated by SIM A. Harder et al. (2010) state that the column value for SIM B $\left(C_{B}\right)$ is varied so that the integrated SIM B irradiance time series (over 200-1600 nm) matches the trend in TSI measured by SORCE TIM. They state that the value of $C_{B}$ is reduced by $4 \%$ as a result of this procedure. Harder [private communication] has stated that the uncertainty in $\kappa(\lambda)$ is approximately $0.6 \%$ based on comparisons of SIM A and SIM B trends at fixed wavelengths.

If we assume that true solar change between May 2004 and November 2007 is consistent with the NRLSSI results shown in Figure 6(b), we can then estimate the magnitude of the error in the SIM calibration terms that would be required to produce the SIM results shown in this figure. Calculating these values for selected UV wavelengths, we find that the value of $\tau(\lambda, t)$ in Eq. [1] would have to be increased by a factor of 1.18 at $240 \mathrm{~nm}$, and increased by a factor of 1.14 at $320 \mathrm{~nm}$. This analysis does not provide further guidance on how such an error might be apportioned between the two terms in Eq. [2]. An error in $\kappa(\lambda)$ might only be important for UV wavelengths, whereas an error in $\mathrm{C}(\mathrm{t})$ would necessarily affect derived irradiance changes for all spectral regions covered by the SIM measurements.

\section{Short-Term Variations.}

Short-term changes in solar UV irradiance arise from the altered distribution of the bright and dark regions on the hemisphere of the Sun visible at Earth due to solar rotation. Faculae represent the dominant influence at shorter UV wavelengths (less than $300 \mathrm{~nm}$ ), while sunspots become more important at wavelengths longer than $300 \mathrm{~nm}$. Lean and Woods (2010) present qualitative comparisons of short-term irradiance variations between NRLSSI model results and 
detrended SIM data over a wide range of spectral bands. The agreement between model and data on this timescale is very good at both faculae-dominated UV wavelengths and sunspotdominated visible wavelengths. TSI values determined by the NRLSSI model also agree with measurements from the SORCE Total Irradiance Monitor (TIM), reproducing both the short term and solar cycle behavior to within the uncertainty of the satellite observations (Kopp and Lean, 2011). These results indicate that the disagreement between the NRLSSI model and the SIM data is only observed over longer time scales in spectral irradiance data, where the accuracy of instrument degradation corrections becomes important. As noted previously, the spectral irradiance results produced by the NRLSSI model are also consistent with previous irradiance measurements on solar cycle time scales.

Rotational variations occur on sufficiently short time scales (approximately 27 days) that instrumental effects in irradiance measurements are much less significant than over solar cycle time scales, and can generally be assumed to be linear over a single rotation. The magnitude of solar irradiance rotational variations at UV wavelengths has a close relationship with short-term $\mathrm{Mg}$ II index variations, which allows the creation of scaling factors to estimate long-term irradiance variations based on $\mathrm{Mg}$ II index behavior. The basic scaling factor method requires the identification of significant rotational variations (defined here as $1 \%$ maximum-to-minimum amplitude) in $\mathrm{Mg}$ II index data. The amplitude of each rotation is calculated using a maximum date and minimum dates before and after the maximum, which averages out any background changes in solar activity that are linear during the rotation. This step is repeated for each irradiance wavelength, thus removing short-term linear instrument changes as well. The set of all rotational amplitudes is then fit using linear regression, and the slope of this function represents the scaling factor for each wavelength. This approach was first demonstrated by 
Heath and Schlesinger (1986), and its accuracy in estimating long-term UV irradiance behavior has been demonstrated in numerous publications (DeLand and Cebula, 1998, 2001, 2008; DeLand et al., 2004; Floyd et al., 2002; Snow et al., 2010). The effectiveness of this twocomponent model (i.e. no additional term for active network changes) has been discussed by Lean et al. (1997). Comparisons with the three-component model of Woods et al. (2000) give consistent results where these models overlap, as discussed by DeLand and Cebula (2008).

We have derived new scale factors for the spectral range 170-300 nm during the period May 2004 - July 2005 using NOAA-17 SBUV/2, UARS SUSIM, SORCE SOLSTICE, and SORCE SIM data, when all four instruments were operating concurrently. While NOAA-17 SBUV/2 data do not have a complete throughput correction in place, the scale factor analysis using rotational amplitudes removes any drift that has a linear time dependence over the $\sim 1$ month time scale of a single solar rotation, as noted previously. The NOAA-17 and SIM data were rebinned to a $1 \mathrm{~nm}$ grid for consistency with the SOLSTICE and SUSIM data sets, since the absolute magnitude of the derived scale factors is dependent on the instrument resolution used for both the Mg II index and the irradiance data. The common measurement time period of May $2004-$ July 2005 yields 12 useful solar rotations, with peak-to-peak amplitudes of $1.3-3.6 \%$ in $\mathrm{Mg}$ II index data. This dynamic range is relatively small for the scale factor analysis, since $\mathrm{Mg}$ II index rotational amplitudes can reach $6-7 \%$ during solar maximum. We therefore included an additional nominal data point representing no change at $[\operatorname{Ratio}(\mathrm{Mg} I I)$, Ratio(irradiance $(\lambda))]=$ $[1.0,1.0]$ to help constrain the regression fit.

The results of the scale factor analysis, using the composite Mg II index from Figure 3(a), are shown in Figure 7. All four instruments produce very similar scale factor results to each other in both absolute value and spectral dependence, indicating a consistent representation of solar 
activity. The spectral variations in Figure 7 are also in excellent agreement with the solar cycle irradiance variations shown in Figure 6(a). The SUSIM results (derived at the end of its 13.5 year lifetime) show more small-scale structure (width $<5 \mathrm{~nm}$ ) than the other instruments, but this behavior does not affect the large-scale spectral dependence. Typical uncertainty values are shown for the NOAA-17 results at $205 \mathrm{~nm}$ and $240 \mathrm{~nm}$. These values are approximately twice as large as the composite scale factor results listed in DeLand and Cebula (1993), in part due to the reduced dynamic range of the rotational data. The slightly lower absolute value of these scale factor results compared to the DeLand and Cebula (1993) results may also be due to the smaller rotational amplitudes available in the selected portion of Cycle 23 , but may also represent real differences between solar cycles. We are investigating this topic in more detail. The results presented in Figure 7 quantitatively support the conclusion of Lean and Woods (2010) that SIM UV measurements observe the same solar physics on short time scales as other observations and model predictions. These results are also in agreement with solar cycle variations determined from both observations and model calculations. This analysis supports our previous conclusion that the discrepancy between SORCE reported long-term UV irradiance results and model prediction is more likely to represent SORCE instrument response correction error.

\section{Terrestrial Response.}

Examination of terrestrial responses as a potential validation of the reported SORCE solar irradiance variations is a complex task because of the non-linear behavior and additional forcing terms in this system. Garcia (2010) has already addressed some of the issues with the results presented by Haigh et al. (2010) for stratospheric effects, and Swartz et al. (2010) has presented additional results based on 3-D atmospheric models. More recently, Merkel et al. (2011) 
presented results of further calculations using the SORCE irradiance variations in the Whole Atmosphere Community Climate Model (WACCM), as well as comparisons of these results with Soundings of the Atmosphere using Broadband Emission Radiometry (SABER) ozone data. They cite a discrepancy between the vertical structure of ozone response to solar forcing produced by WACCM (forced with NRLSSI inputs) and SABER data during 2004-2007 as evidence for problems with the NRLSSI solar variations. However, many previous authors have noted this difference between models and observations (e.g. Austin et al., 2008; Soukharev and Hood, 2006), using data sets back to 1978. Meanwhile, the vertical distribution of ozone in the tropical stratosphere produced by WACCM with SORCE forcing differs significantly from both the NRLSSI-forced model result and SABER data.

Merkel et al. (2011) also present multiple regression analysis to estimate the solar contribution to trends in the SABER data in the stratosphere $(5.0-1.2 \mathrm{hPa})$ and lower mesosphere (0.3-0.03 hPa). Their analysis covers the SABER data record from July 2002 to December 2010 , although SIM data provided for this paper only extend from April 2004 to January 2009. Further extension of solar UV activity back to 2002 by Merkel et al. (2011) was done based on Ca II K images from the Precision Solar Photometric Telescope (PSPT) (Criscuoli et al., 2010). However, there is no description of how these images are quantitatively related to spectral UV irradiance variations that directly affect the stratosphere and lower mesosphere. The referenced modeling paper by Fontenla and Harder (2005) only addresses the visible and IR spectral regions. Thus, it is impossible to assess whether the solar cycle UV variations used by Merkel et al. (2011) are consistent with UV irradiance observations made by UARS SUSIM during 20022005. 


\section{Conclusion.}

The temporal and spectral dependence of solar UV irradiance variations over a solar cycle has been well-established by almost 30 years of satellite measurements. Model representations using both proxy indexes and surface magnetic features accurately reproduce these observations. SORCE SIM and SORCE SOLSTICE measurements of solar UV irradiance during the declining phase of Cycle 23 show large changes that are inconsistent with these previous results and concurrent proxy index data, and also show spectral variations that differ from solar physics theory. However, short-term SIM and SOLSTICE irradiance variations do yield good agreement with concurrent solar UV data from other instruments. Rather than requiring an unprecedented change in how the Sun works, we feel that the most likely explanation for these results is an incomplete characterization of SORCE on-orbit instrument degradation effects. Additional validation of these SORCE observations is crucial to prevent a future research path based on a foundation of unrealistic solar-terrestrial behavior.

\section{Acknowledgements}

Jerry Harder provided the SORCE SIM ultraviolet irradiance data set used in this paper. Luca Bertello provided the conversion formula to create $\mathrm{Ca}$ II $\mathrm{K}$ index values from MPSI data. This study includes data from the synoptic program at the 150-Foot Solar Tower of the Mt. Wilson Observatory. The Mt. Wilson 150-Foot Solar Tower is operated by UCLA, with funding from NASA, ONR, and NSF, under agreement with the Mt. Wilson Institute. The raw SBUV/2 data were obtained from NOAA/NESDIS with support from the NOAA Climate and Global Change Atmospheric Chemistry Element. This work was supported by NASA through contract NNH06HX18C. 


\section{References}

Ahmad, Z., DeLand, M. T., Cebula, R. P., Weiss, H., Wellemeyer, C. G., Planet, W. G., Lienesch, J. H., Bowman, H. D., Miller, A. J., Nagatani, R. M., 1994. Accuracy of total ozone retrieval from NOAA SBUV/2 measurements: Impact of instrument performance. J. Geophys. Res. 99, 22,975-22,984.

Austin, J., et al., 2008. Coupled chemistry climate model simulations of the solar cycle in ozone and temperature. J. Geophys. Res. 113, D11306, doi:10.1029/2007JD009391.

Ball, W. T., Unruh, Y. C., Krivova, N. A., Solanki, S., Harder, J. W., 2011. Solar irradiance variability: a six-year comparison between SORCE observations and the SATIRE model. Astron. Astrophys. 530, A71, doi:10.1051/0004-6361/201016189.

Bertello, L., Ulrich, R. K., Boyden, J. E., 2010. The Mount Wilson Ca II K plage index time series. Solar Phys. 264, 31-44.

Cebula, R. P., DeLand, M. T., 1998. NOAA 11 Solar Backscatter Ultraviolet, model 2 (SBUV/2) instrument solar spectral irradiance changes in 1989-1994. 1: Observations and long-term calibration. J. Geophys. Res. 103, 16,235-16,249.

Cebula, R. P., Thuillier, G. O., VanHoosier, M. E., Hilsenrath, E., Herse, M., Brueckner, G. E., Simon, P. C., 1996. Observations of the solar irradiance in the 200-350 $\mathrm{mm}$ interval during the ATLAS-1 mission: a comparison among three sets of measurements SSBUV, SOLSPEC, and SUSIM. Geophys. Res. Lett. 23, 2289-2292.

Criscuoli, S., Ermolli, I., Fontenla, J., Giorgi, F., Rast, M., Solanki, S. K., Uitenbroek, H., 2010. Radiative emission of solar features in Ca II K. Mem. Soc. Astron. Ital. 81, 773-776.

DeLand, M. T., Cebula, R. P., 1993. Composite Mg II solar activity index for solar cycles 21 and 22. J. Geophys. Res. 98, 12,809-12,823.

DeLand, M. T., Cebula, R. P., 1998. NOAA 11 Solar Backscattered Ultraviolet, model 2 (SBUV/2) instrument solar spectral irradiance measurements in 1989-1994, 2. Results, validation, and comparisons. J. Geophys. Res. 103, 16,251-16,273.

DeLand, M. T., Cebula, R. P., 2001. Spectral solar UV irradiance data for cycle 21. J. Geophys. Res. 106, 21,569-21,583.

DeLand, M. T., Cebula, R. P., 2008. Creation of a composite solar ultraviolet spectral irradiance data set. J. Geophys. Res. 113, A11103, doi:10.1029/2008JA013401. 
DeLand, M. T., Cebula, R. P., Hilsenrath, E., 2004. Observations of solar spectral irradiance change during cycle 22 from NOAA-9 Solar Backscattered Ultraviolet Model 2 (SBUV/2). J. Geophys. Res. 109, D06304, doi:10.1029/2003JD004074.

Floyd, L. E., Herring, L. C., Prinz, D. K., Crane, P. C., 1998. Instrument responsivity evolution of SUSIM UARS. Proc. SPIE 3427, 445-456.

Floyd, L. E., Prinz, D. K., Crane, P. C., Herring, L. C., 2002. Solar UV irradiance variation during cycles 22 and 23. Adv. Space Res. 29, 1957-1962.

Floyd, L. E., Cook, J. W., Herring, L. C., Crane, P. C., 2003. SUSIM's 11-year observational record of the solar UV irradiance. Adv. Space Res. 31, 2111-2120.

Fontenla, J., Harder, G., 2005. Physical modeling of spectral irradiance variations. Mem. Soc. Astron. Ital. 76, 826-833.

Foukal, P., 1990. Solar Astrophysics, John Wiley and Sons, New York.

Garcia, R. R., 2010. Solar surprise? Nature 467, 668-669.

Haigh, J. D., Winning, A. R., Toumi, R., Harder, J. W., 2010. An influence of solar spectral variations on radiative forcing of climate. Nature 467, 696-699.

Harder, J., Lawrence, G., Fontenla, J., Rottman, G., Woods, T. Woods, 2005. The Spectral Irradiance Monitor: Scientific requirements, instrument design, and operation modes. Solar Phys. 230, 141-167.

Harder, J. W., Fontenla, J. M., Pilewskie, P., Richard, E. C., Woods, T. N., 2009. Trends in solar spectral irradiance variability in the visible and infrared. Geophys. Res. Lett. 36, L07801, doi:10.1029/2008GL036797.

Harder, J. W., Thuillier, G., Richard, E. C., Brown, S. W., Lykke, K. R., Snow, M., McClintock, W. E., Fontenla, J. M., Woods, T. N., Pilewskie, P., 2010. The SORCE SIM solar spectrum: Comparison with recent observations. Solar Phys. 263, 3-24.

Heath, D. F., 1988. Non-seasonal changes in total column ozone from satellite observations, 1970-1986. Nature 332, 219-227.

Heath, D. F., Schlesinger, B. M., 1986. The Mg-280 nm doublet as a monitor of changes in solar ultraviolet irradiance. J. Geophys. Res. 91, 8672-8682.

Herman, J. R., Hudson, R. D., Serafino, G., 1990. Analysis of the eight-year trend in ozone depletion from empirical models of Solar Backscatter Ultraviolet instrument degradation. J. Geophys. Res. 95, 7403-7416. 
Hilsenrath, E., Cebula, R. P., DeLand, M. T., Laamann, K., Taylor, S., Wellemeyer, C., Bhartia, P. K., 1995. Calibration of the NOAA 11 solar backscatter ultraviolet (SBUV/2) ozone data set from 1989 to 1993 using in-flight calibration data and SSBUV. J. Geophys. Res. $100,1351-1366$.

Kopp, G., Lean, J. L., 2011. A new, lower value of total solar irradiance: Evidence and climate significance. Geophys. Res. Lett. 38, L01706, doi:10.1029/2010GL045777.

Lean, J. L., Woods, T. N., 2010. Solar Spectral Irradiance Measurements and Models, in: Schrijver, K., Siscoe, G. (Eds.), Evolving Solar Physics and the Climates of Earth and Space, Cambridge Univ. Press, London.

Lean, J. L., Rottman, G. J., Kyle, H. L., Woods, T. N., Hickey, J. R., Puga, L. C., 1997. Detection and parameterization of variations in solar mid and near ultraviolet radiation (200 to $400 \mathrm{~nm}$ ). J. Geophys. Res. 102, 29,939-29,956.

Lean, J., Rottman, G., Harder, J., Kopp, G., 2005. SORCE contributions to new understanding of global change and solar variability. Solar Phys. 230, 27-53.

Lockwood, M., 2011. Was UV spectral solar irradiance lower during the recent low sunspot minimum? J. Geophys. Res. 116, D16103, doi:10.1029/2010JD014746.

Lockwood, M., Bell, C., Woollings, T., Harrison, R. G., Gray, L. J., Haigh, J. D., 2010. Topdown solar modulation of climate: evidence for centennial-scale change. Environ. Res. Lett. 5, doi:10.1088/1748-9326/5/3/034008.

McClintock, W. E., Snow, M., Woods, T. N., 2005. Solar Stellar Irradiance Comparison Experiment II (SOLSTICE II): Prelaunch and on-orbit calibration. Solar Phys. 230, 259294.

Meier, R. R., 1991. Ultraviolet spectroscopy and remote sensing of the upper atmosphere. Space Sci. Rev. 58, 1-185.

Merkel, A. W., Harder, J. W., Marsh, D. R., Smith, A. K., Fontenla, J. M., Woods, T. N., 2011. The impact of solar spectral irradiance variability on middle atmospheric ozone. Geophys. Res. Lett. 38, L13802, doi:10.1029/2011GL047561.

Rottman, G. J., 1988. Observations of solar UV and EUV variability. Adv. Space Res. 8(7), 5366.

Rottman, G., Woods, T., Snow, M., DeToma, G., 2001. The solar cycle variation in ultraviolet irradiance. Adv. Space Res. 27, 1927-1932.

Snow, M., McClintock, W. E., Rottman, G., Woods, T. N., 2005. Solar stellar irradiance comparison experiment II (SOLSTICE II): Examination of the solar-stellar calibration technique. Solar Phys. 230, 295-324. 
Snow, M., McClintock, W. E., Woods, T. N., 2010. Solar spectral irradiance variability in the ultraviolet from SORCE and UARS SOLSTICE. Adv. Space Res. 46, 296-302.

Soukharev, B. E., Hood, L. L., 2006. Solar cycle variation of stratospheric ozone: Multiple regression analysis of long-term satellite data sets and comparisons with models. J. Geophys. Res. 111, D20314, doi:10.1029/2006JD007107.

Swartz, W. H., Stolarski, R. S., Oman, L., Fleming, E. L., Jackman, C. H., 2010. Solar cycle effects of spectrally varying solar irradiance in a coupled chemistry-climate model. Eos Trans. AGU 91, paper GC13E-03.

Viereck, R. A., Floyd, L. E., Crane, P. C., Woods, T. N., Knapp, B. G., Rottman, G., Weber, M., Puga, L. C., DeLand, M. T., 2004. A composite Mg II index spanning from 1978 to 2003. Space Weather 2, S10005, doi:10.1029/2004SW000084.

Woods, T. N., Tobiska, W. K., Rottman, G. J., Worden, J. R., 2000. Improved solar Lyman $\alpha$ irradiance modeling from 1947 through 1999 based on UARS observations. J. Geophys. Res. 105, 27,195-27,215. 


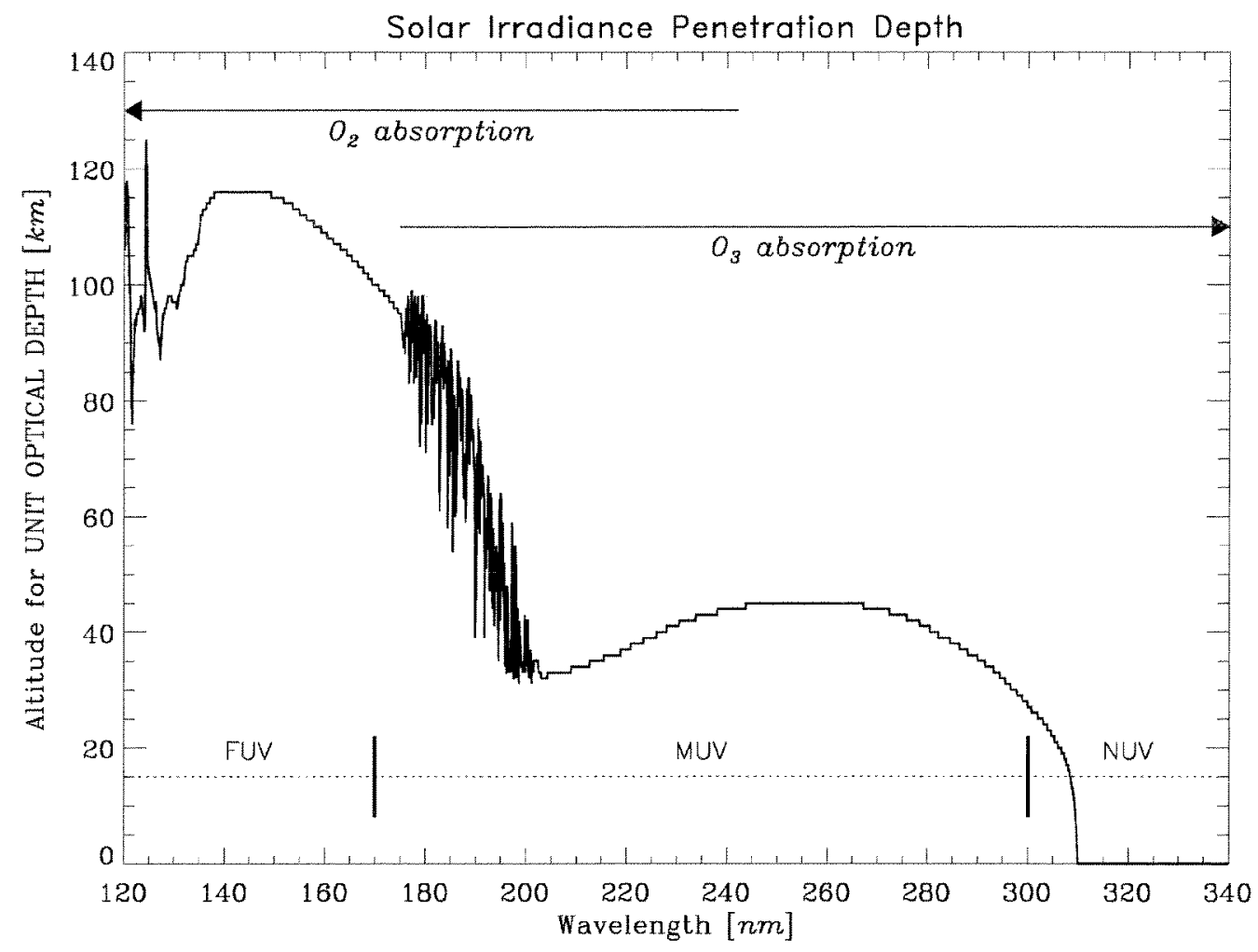

Figure 1. Depth in the atmosphere at which incoming solar radiation is attenuated by a factor of 1/e (optical depth $\tau=1$ ). Specific wavelength regions relevant to this paper are: far ultraviolet $(\mathrm{FUV}=120-170 \mathrm{~nm})$, mid-ultraviolet $(\mathrm{MUV}=170-300 \mathrm{~nm})$, near-ultraviolet $(\mathrm{NUV}=300-400$ nm). Adapted from Meier (1991). 


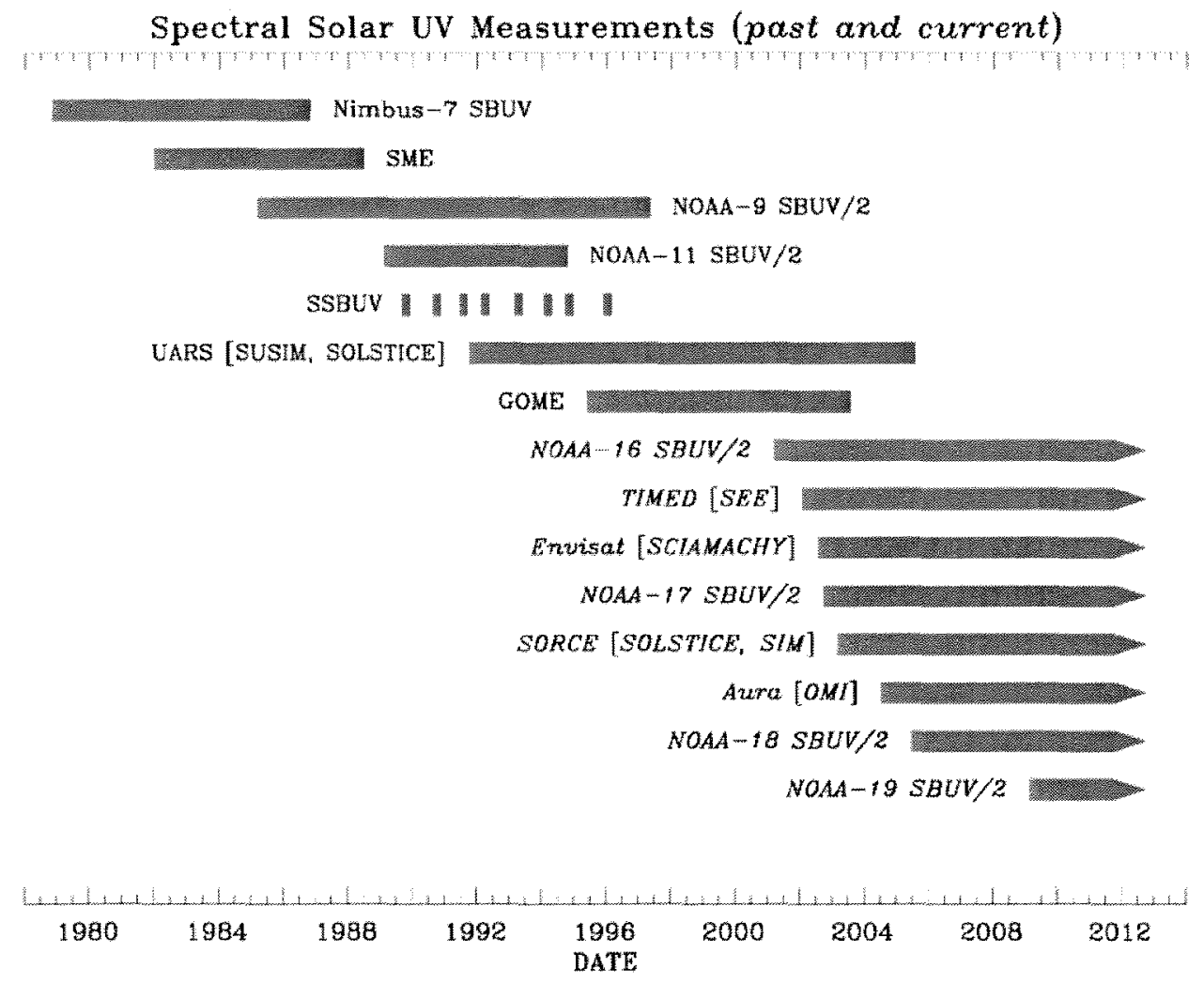

Figure 2. Timeline of solar UV spectral irradiance measurements. Active instruments as of September 2011 are shown with arrowheads. 

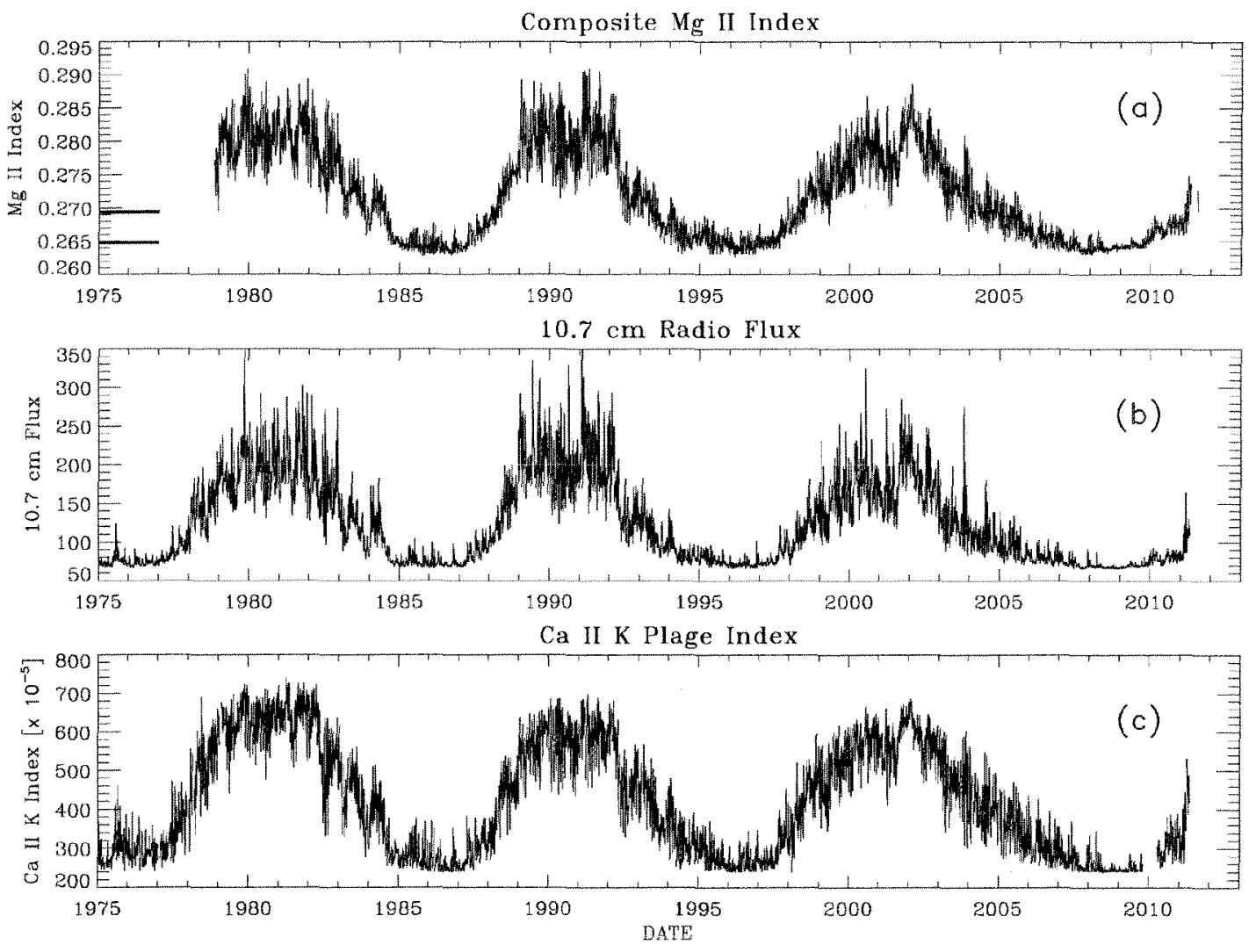

Figure 3. Solar UV proxy time series. (a) Composite Mg II index time series produced by Viereck et al. (2004), extended using SORCE SOLSTICE Mg II index values (Snow et al., 2010). The $\mathrm{Y}$-axis tick marks indicate the level of solar activity corresponding to May 2004 and November 2007, the dates used by Haigh et al. (2010). (b) $10.7 \mathrm{~cm}$ radio flux time series from NOAA National Geophysical Data Center (NGDC). (c) Ca II K plage index values produced from Mt. Wilson magnetic plage strength index (MPSI) data by Bertello et al. (2010) and extended through April 2011. 

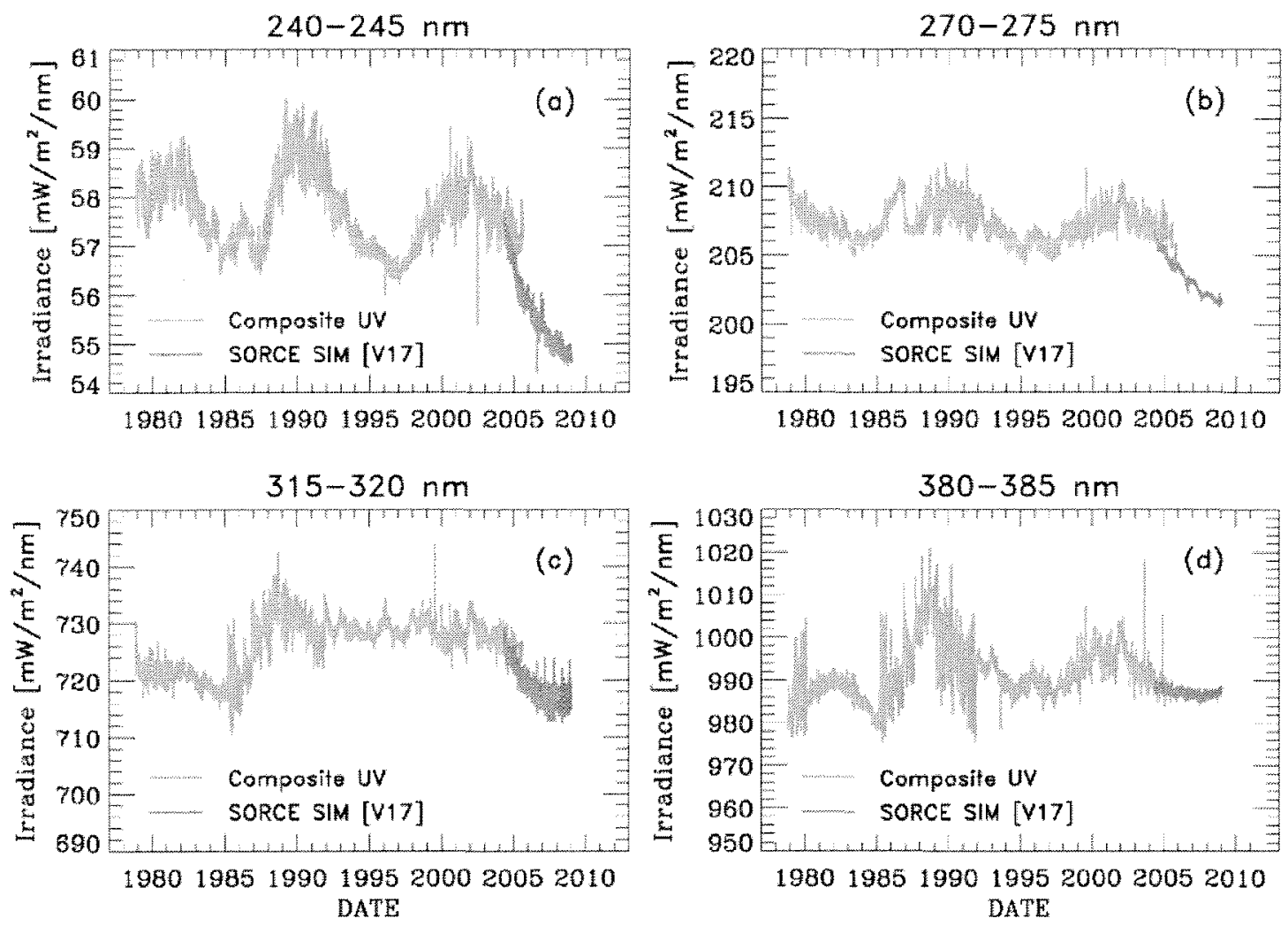

Figure 4. SORCE SIM (orange) and composite UV irradiance (green) data for four spectral bands. The SIM data are normalized to match the composite UV data in April 2004. Composite UV data between October 1986 and February 1989 have been shifted upwards by $+1.2 \%$, consistent with the analysis of Lockwood et al. (2010). Panels: (a) $=240-245 \mathrm{~nm},(\mathrm{~b})=270-275$ $\mathrm{nm},(\mathrm{c})=315-320 \mathrm{~nm},(\mathrm{~d})=380-385 \mathrm{~nm}$. 

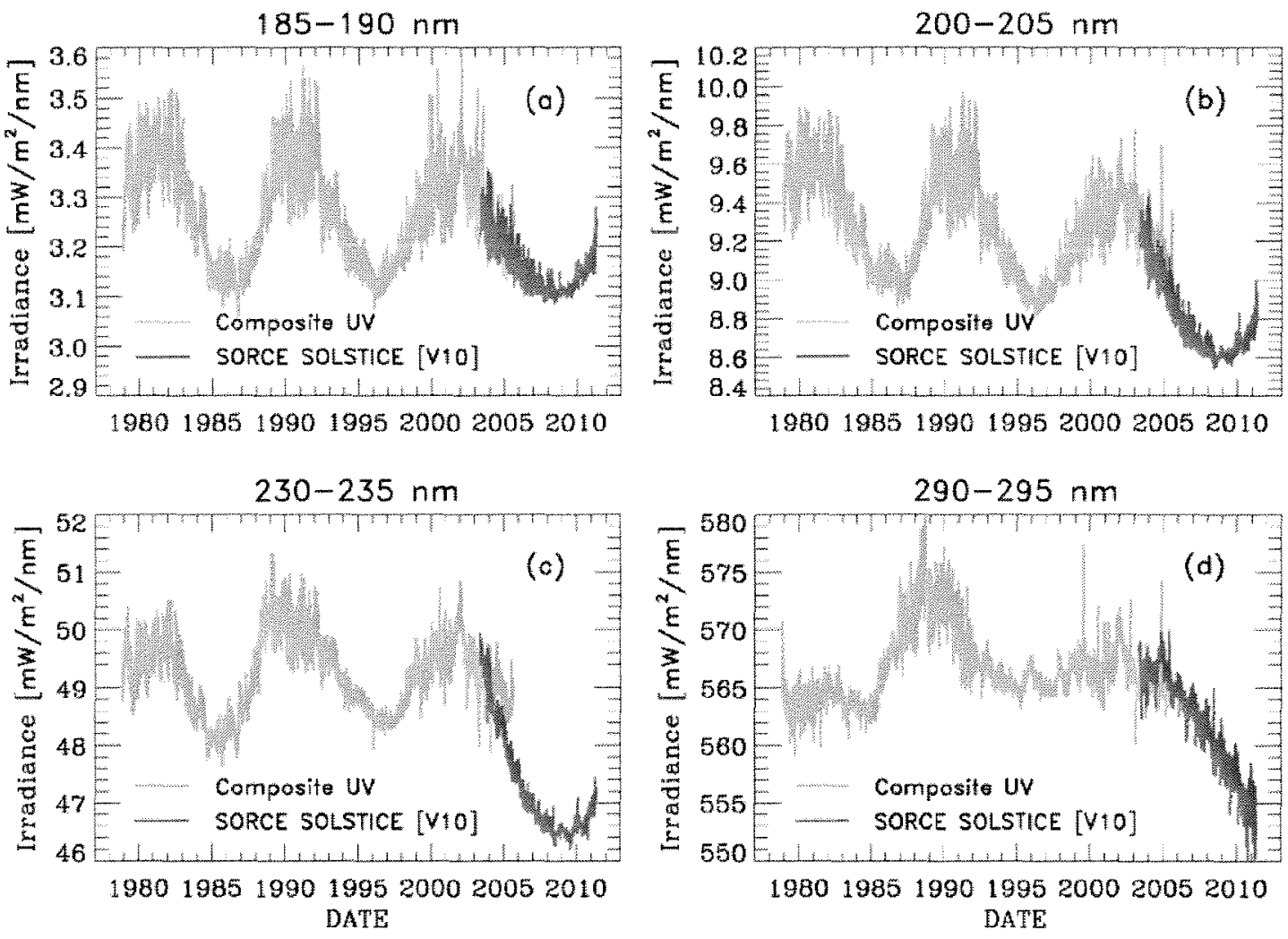

Figure 5. SORCE SOLSTICE (red) and composite UV irradiance (green) data for four spectral bands. The SOLSTICE data are normalized to match the composite UV data in September 2003. Composite UV data between October 1986 and February 1989 have been shifted upwards by $+1.2 \%$, consistent with the analysis of Lockwood et al. (2010). Panels: (a) $=185-190 \mathrm{~nm}$, (b) $=$ $200-205 \mathrm{~nm},(\mathrm{c})=230-235 \mathrm{~nm},(\mathrm{~d})=290-295 \mathrm{~nm}$. 

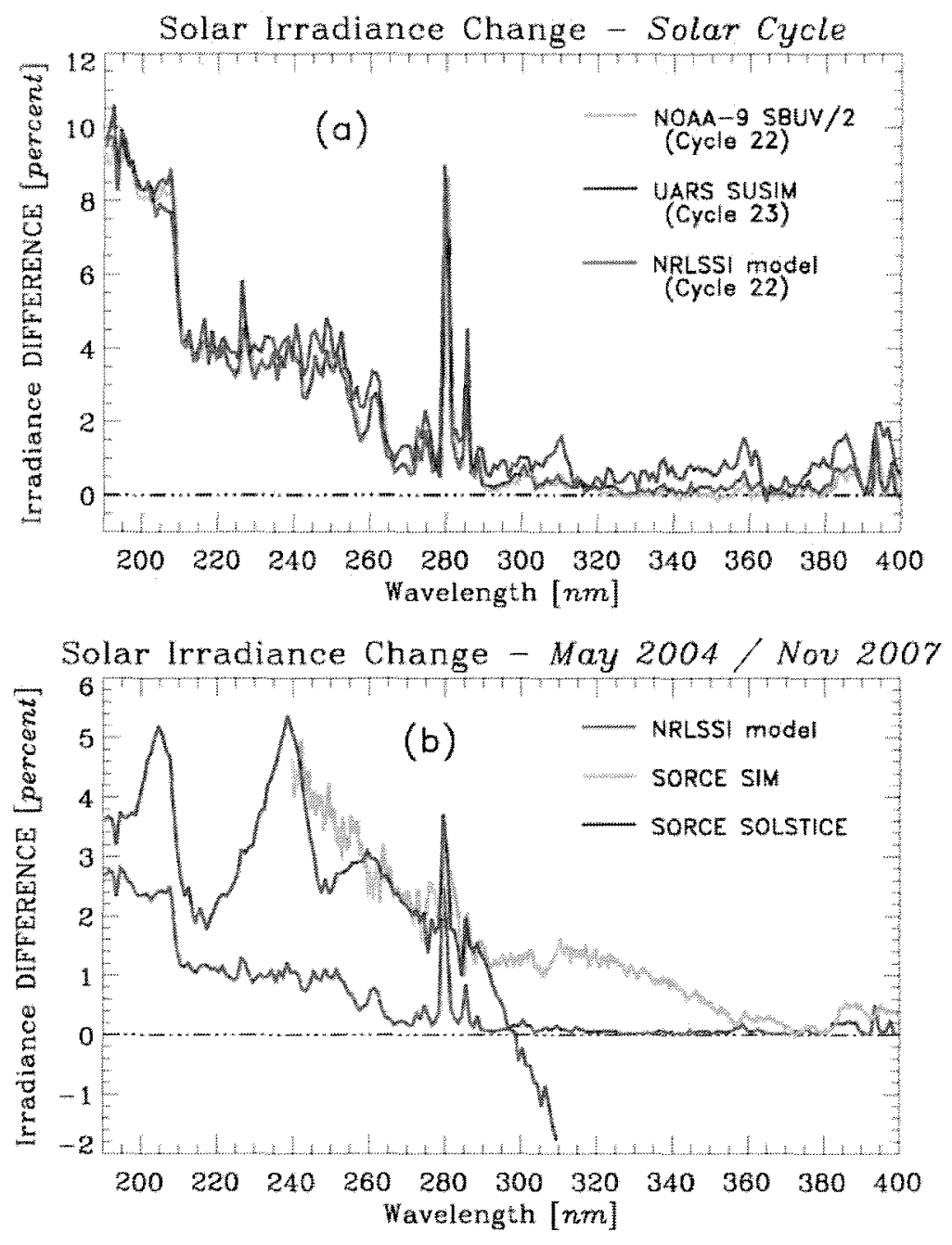

Figure 6. (a) Solar cycle irradiance change between 190-400 nm (in percent). All data are binned to $1 \mathrm{~nm}$ sampling. The NOAA-9 SBUV/2 (green), UARS SUSIM (blue), and NRLSSI (red) curves represent monthly average solar cycle maximum to minimum variations. (b) Solar irradiance change between 190-400 nm (in percent) from May 2004 to November 2007 for the NRLSSI model (red), SORCE SIM data (green), and SORCE SOLSTICE data (blue). All curves represent comparisons of monthly average irradiance spectra. 


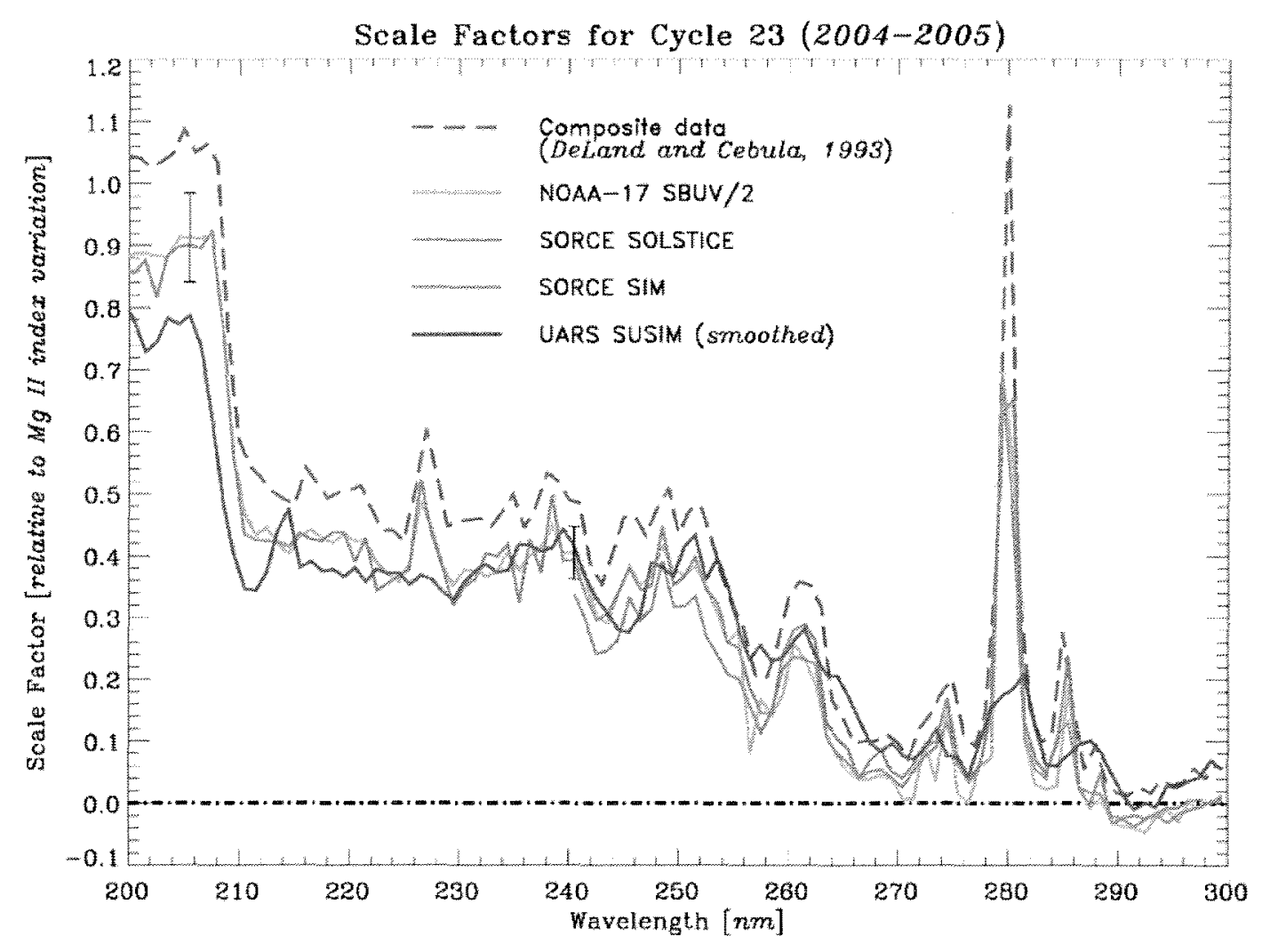

Figure 7. Scale factors for Mg II index variations derived from irradiance measurements during the descending phase of solar cycle 23. All results are shown for $1 \mathrm{~nm}$ gridded irradiance data. The SIM results are limited to $240-300 \mathrm{~nm}$. The SUSIM results have been smoothed with a 5 point boxcar average to remove small-scale structure. The uncertainty of the derived NOAA-17 scale factors is shown at $205 \mathrm{~nm}$ and $240 \mathrm{~nm}$. The composite scale factors from DeLand and Cebula (1993), derived from Cycle 21-22 data, are shown for reference. 